\title{
COMMENTARY
}

\section{New targets for new therapeutic approaches}

\author{
Bruno François ${ }^{1,2}$ \\ See related review by Sawa et al., http://ccforum.com/content/18/6/668
}

\begin{abstract}
Because of its resistance profiles, Pseudomonas aeruginosa remains probably one of the challenging bacteria responsible for ventilator-associated pneumonia in the ICU. Nevertheless, a much better understanding of its mechanism of virulence, such as the type 3 secretion system that can also impact on resistance, gives some opportunities for management improvement. The most promising approach is probably the production of monoclonal antibodies that enable not only more targeted treatments but also development of some early preemptive approaches at the time of colonization through real-time diagnosis.
\end{abstract}

Ventilator-associated pneumonia (VAP) is probably one of the last infections remaining challenging in the ICU, unlike sepsis and other ICU-specific infections for which management is much better defined, and Sawa and colleagues bring some new understanding and therapeutic options [1]. In this specific clinical setting, two microorganisms are predominantly involved - Staphylococcus aureus and Pseudomonas aeruginosa - both sharing the same resistance issues and treatment controversies.

Owing to its larger genome, encoding extreme phenotypic versatility, motility, virulence, and persistence factors as well as its intrinsic and acquired drug resistance, $P$. aeruginosa is possibly the most interesting of the opportunistic bacteria. In this issue of Critical Care, Sawa and colleagues demonstrate that $P$. aeruginosa has the capability of producing numerous toxins, of which ExoU seems to play a key role. These authors convincingly illustrate the potential link between $P$. aeruginosa virulence and resistance, with a special emphasis on ExoU-associated virulence. As a major component of the type 3 secretion system (T3SS), which is responsible for the injection of

Correspondence: b.francois@unilim.fr

'Service de Réanimation Polyvalente, CHU de Limoges, 2 Avenue Martin Luther King, 87042 Limoges, Cedex, France

${ }^{2}$ INSERM, CIC 1435, CHU Dupuytren, 2 avenue Martin Luther King, 87042 Limoges, Cedex, France

() Biomed Central
ExoU and various cytotoxic virulence factors into host cells, PcrV appears to be a promising target for future VAP prevention agents. Importantly, clinical studies have previously suggested a relationship between $P$. aeruginosa T3SS gene expression in clinical isolates (blood cultures) and outcome [2]. Additional virulence factors are also frequently involved in P. aeruginosa VAP. Quorum sensingregulated virulence factor rhamnolipids have been identified in patients with $P$. aeruginosa VAP and its inhibition appears to be an alternative therapeutic strategy [3]. Psl is a serotype-independent exopolysaccharide implicated in initial colonization and so-called immune evasion [4]. Of note, these various mechanisms of virulence/resistance may interact, such as Psl and T3SS expression, and jointly P. aeruginosa infections, including VAP [5].

While the $P$. aeruginosa mechanism of action is increasingly decrypted, diagnostic methods have tremendously improved over the last decade, moving from timeconsuming conventional culture towards real-time bedside diagnosis. Reverse transcription polymerase chain reaction is bringing online diagnosis to the clinical grounds and promises to enable early targeted therapy in the near future. In addition, continuous technical proceedings facilitate analysis processes and open new perspectives on the routine use of these novel diagnostic approaches for a bedside analysis directly on any lung biological sample (bronchoalveolar lavage, endotracheal aspirates, and so forth). Undoubtedly, $P$. aeruginosa virulence determinants being better understood, these newly developed diagnostic techniques promise to simultaneously provide the frontline intensivist with valuable information on bacterial virulence and even the drug-resistance profile.

In parallel, VAP management has also improved dramatically. The major issue of induced resistance by antibiotics definitely supports the recommended bundles to prevent VAP, but other benefits are also the prioritization of targeted antibiotic therapy and reduced treatment duration [6]. The most promising therapeutic perspective is the identification of specific $P$. aeruginosa targets for specific monoclonal antibody $(\mathrm{mAb})$ development. 
Several anti-pseudomonas mAbs are currently developed for clinical use and recent publications have demonstrated their potential clinical value for VAP treatment $[7,8]$. The combined early $P$. aeruginosa infection diagnosis capability using real-time techniques such as polymerase chain reaction and $\mathrm{mAb}$ availability have paved a new road for the preemptive therapeutic approach in $P$. aeruginosa VAP and introduced a paradigm shift in critical care medicine. With colonization preceding infection (also sometimes referred to as disease) in nearly all cases, the challenge is now to confirm the validity of such an approach. We reported recently convincing results in a phase II trial that tested a preemptive mAb therapeutic approach targeting PcRv in ICU $P$. aeruginosa colonized patients [8]. In addition, given their complementary roles, a combination of the anti-Psl and anti-PcrV mAbs as a single drug candidate (MEDI3902; MedImmune, AstraZeneca, Gaithersburg, MD, USA) could increase the benefit in a preemptive VAP approach against $P$. aeruginosa. To test this hypothesis, trials should start soon within the ND4BB program (supported by the Innovative Medicines Initiative) that made the fight against multiresistant bacteria, and especially $P$. aeruginosa, one of its main objectives. A similar preemptive approach targeting $S$. aureus alpha toxin with a long half-life mAb (MEDI4893; MedImmune, AstraZeneca) is already underway in this program.

In conclusion, it is probably not unrealistic to think that in the very near future we should be able to rapidly detect the presence of $P$. aeruginosa in the lung or lower respiratory tract and at the same time assess both drug-resistance and virulence potential for a preemptive strategy using highly specific mAbs to neutralize key targets in bacterial virulence, persistence and resistance to therapy. This theranostic approach focused on new targets could undoubtedly be the magic bullet to reduce VAP incidence, as well as the VAP-associated morbidity and mortality.

\section{Abbreviations}

mAb: monoclonal antibody; T3SS: type 3 secretion system;

VAP: ventilator-associated pneumonia.

\section{Competing interests}

The author declares that he has no competing interests.

Published online: 13 December 2014

\section{References}

1. Sawa T, Shimizu M, Moriyama K, Wiener-Kronish J: Association between Pseudomonas aeruginosa type III secretion, antibiotic resistance, and clinical outcome: a review. Crit Care 2014.

2. El-Solh AA, Hattemer A, Hauser AR, Alhajhusain A, Vora H: Clinical outcomes of type III Pseudomonas aeruginosa bacteremia. Crit Care Med 2012, 40:1157-1163.

3. Van Delden $C$, Köhler $T$, Brunner-Ferber F, François B, Carlet J, Pechère JC: Azithromycin to prevent Pseudomonas aeruginosa ventilator-associated pneumonia by inhibition of quorum sensing: a randomized controlled trial. Intensive Care Med 2012, 38:1118-1125.
4. Jackson KD, Starkey M, Kremer S, Parsek MR, Wozniak DJ: Identification of psl, a locus encoding a potential exopolysaccharide that is essential for Pseudomonas aeruginosa PAO1 biofilm formation. J Bacteriol 2004, 186:4466-4475.

5. Mikkelsen $H$, Sivaneson M, Filloux A: Key two-component regulatory systems that control biofilm formation in Pseudomonas aeruginosa. Environ Microbiol 2011, 13:1666-1681.

6. Dimopoulos G, Poulakou G, Pneumatikos IA, Armaganidis A, Kollef MH, Matthaiou DK: Short- vs long-duration antibiotic regimens for ventilator-associated pneumonia: a systematic review and meta-analysis. Chest 2013, 144:1759-1767.

7. Que YA, Lazar H, Wolff M, François B, Laterre PF, Mercier E, Garbino J, Pagani JL, Revelly JP, Mus E, Perez A, Tamm M, Rouby JJ, Lu Q, Chastre J, Eggimann P: Assessment of panobacumab as adjunctive immunotherapy for the treatment of nosocomial Pseudomonas aeruginosa pneumonia. Eur J Clin Microbiol Infect Dis 2014, 33:1861-1867.

8. François B, Luyt CE, Dugard A, Wolff M, Diehl JL, Jaber S, Forel JM, Garot D, Kipnis E, Mebazaa A, Misset B, Andremont A, Ploy MC, Jacobs A, Yarranton G, Pearce T, Fagon JY, Chastre J: Safety and pharmacokinetics of an anti-PcrV PEGylated monoclonal antibody fragment in mechanically ventilated patients colonized with Pseudomonas aeruginosa: a randomized, double-blind, placebo-controlled trial. Crit Care Med 2012, 40:2320-2326.

doi:10.1186/s13054-014-0669-8

Cite this article as: François: New targets for new therapeutic approaches. Critical Care 2014 18:669. 